\title{
Desarrollo de un cuestionario de actitudes hacia la donación de órganos postmortem ${ }^{1}$
}

\section{Development of a attitude questionnaire toward post-mortem organ donation}

\author{
Kristian Jasso' ${ }^{2}$, Roberto Lagunes-Córdoba², \\ José Manuel Martínez y María Luisa Marván ${ }^{2}$
}

\begin{abstract}
RESUMEN
La disposición para donar órganos está influida por diversos factores psicosociales, entre los que se encuentran las actitudes hacia la donación. El objetivo del presente artículo fue diseñar y validar un cuestionario de actitudes respecto a la donación de órganos postmortem (ADOP). El instrumento se validó en una muestra de 1,029 adultos mexicanos de diferentes edades y niveles educativos. Se evaluó la validez de contenido mediante dos consultas a expertos (jueceo cualitativo y cuantitativo), la validez de constructo por medio del análisis factorial (exploratorio y confirmatorio) y la confiabilidad con el coeficiente alfa de Cronbach. El ADOP quedó conformado por tres factores: Actitudes favorables, Actitudes desfavorables y Actitudes de desconfianza. El coeficiente alfa de Cronbach fue de 0.87 , lo que indica una buena confiabilidad. Se concluye que el ADOP posee propiedades psicométricas aceptables, así como una estructura clara e interpretable.
\end{abstract}

Palabras clave: Donación de órganos; Actitudes; Validación de cuestionarios.

\begin{abstract}
Willingness to donate organs is influenced by diverse psychosocial factors, including beliefs and attitudes towards organ donation itself. The objective of the present study was to develop and validate a questionnaire measuring attitudes toward posthumous organ donation (APOD). The instrument was validated with 1,029 Mexican adults of different ages and educational levels. Content validity was assessed through two expert consultations (qualitative and quantitative). Construct validity was established through exploratory and confirmatory factor analysis, and internal consistency through Cronbach's alpha coefficient. The APOD contains three factors: Favorable attitudes, Unfavorable attitudes, and Attitudes of mistrust. The instrument showed a Cronbach's alpha of 0.87, indicating good internal consistency. The APOD has acceptable psychometric properties, as well as an easy to interpret and relevant factor structure.
\end{abstract}

Key words: Organ donation; Attitudes; Questionnaires validation.

$\mathrm{L}$

os trasplantes de órganos posibilitan salvar, o mejorar, la vida de muchos pacientes. Una persona puede ser donante en vida; cabe mencionar que en la donación postmortem (o cadavérica) es posible obtener más órganos que en la donación en vida, la que además conlleva ciertos riesgos para el donante (World Health Organization [WHO], 2017). Por ello, el presente estudio se centró en las actitu-

\footnotetext{
${ }^{1}$ Los autores agradecen a Fabiola Orihuela y a María Fernanda Marván por su ayuda en la recolección de datos.

${ }^{2}$ Instituto de Investigaciones Psicológicas, Universidad Veracruzana, Av. Dr. Luis Castelazo Ayala s/n, Col. Industrial Ánimas, 91190 Xalapa, Ver., México, tel. (228)841-89-00, ext. 13210, correo electrónico: mlmarvan@gmail.com.

${ }^{3}$ Departamento de Psicología Social y Metodología, c/Ivan Pavlov no 6, Ciudad Universitaria de Cantoblanco, Universidad Autónoma de Madrid, 28049 Madrid, España, tel. (34)914-97-51-00.

Citación: Jasso, K., Lagunes-Córdoba, R., Martínez, J.M. y Marván, M.L. (2018). Desarrollo de un cuestionario de actitudes hacia la donación de órganos postmortem. Psicología y Salud, 28(2), 195-205.
} 
des que se observan hacia la donación postmortem. Hay que señalar que para que este tipo de donación pueda llevarse a cabo es necesario que los médicos tratantes certifiquen el diagnóstico de muerte a partir de uno de dos criterios posibles: muerte cardiorrespiratoria o muerte cerebral, siendo esta última la que permite la donación de más órganos (WHO, 2009). Luego del fallecimiento, es necesario que la familia autorice la donación, y entonces se puede proceder a la extracción de los órganos y tejidos para su trasplante.

Desafortunadamente, la demanda de órganos para trasplante supera la oferta, y por ello muchas personas fallecen en la espera del órgano que requieren (Findlater y Thomson, 2015). España es el líder mundial en donación de órganos, y en 2015 tuvo una tasa de 40.2 donadores por cada millón de habitantes (pmh). En contraste, en ese mismo año México tuvo una tasa de 3.4 donadores pmh, cifra inferior al promedio de los países latinoamericanos, misma que fue de 6.51 pmh (Council of Europe, 2016). El Global Observatory on Donation and Trasplantation clasificó a los países de los que se tienen datos oficiales en seis grupos según su tasa de donadores; en tal clasificación México se encuentra en el grupo que tiene las tasas más bajas.

Breitkopf (2009) hizo un análisis de los estudios publicados que se llevaron a cabo en comunidades de hispanos que residen en Estados Unidos, concluyendo que estos, al ser comparados, con la población caucásica, son menos propensos a donar sus órganos debido a una variedad de factores relacionados con el miedo, la ignorancia o los prejuicios religiosos. Por ejemplo, algunos de esos estudios señalan que muchos hispanos creen que la donación se lleva a cabo antes de la muerte, lo que puede explicarse porqué existe confusión sobre el concepto de muerte cerebral. Otras barreras encontradas en estas comunidades fueron el deseo de ser sepultados con todas las partes de su cuerpo intactas, así como la idea de que la religión católica se opone a la donación y el trasplante de órganos, a pesar de que ninguna religión los prohíbe formalmente (Randhawa y Neuberger, 2016); incluso la Iglesia católica alienta a la donación. En diversos estudios llevados a cabo en diferentes países de América Latina se han señalado obstáculos semejantes a los descritos, y también otros, como la creencia de que las personas de edad avanzada no pueden donar órganos; que el receptor de los órganos adopta los gustos, sentimientos y rasgos de personalidad de su donador, y que la donación implica la mutilación o la desfiguración del cuerpo (Bustamante y Villarreal, 2008; Gómez, Ballena y León, 2016; Lozano, Liberman, Ito y Andrade, 2005; Zepeda, García y Aguirre, 2003). Algunas de esas barreras también se han reportado en otras regiones del mundo, en unas con más frecuencia que en otras (Bruzzone, 2008; Güden, Çetinkaya y Naçar, 2013; Wong, 2011).

Otras razones por las que algunas personas no están dispuestas a donar sus órganos tienen que ver con la desconfianza. Se ha reportado esa falta de confianza en el procedimiento médico en sí, aunque es más frecuente la creencia de que hay ineficiencia o deshonestidad en el manejo del proceso de trasplante de órganos (Lozano et al., 2005; Marván, Álvarez, Jasso y Santillán, 2017; Weiss, 2003). De hecho, hay quienes han comentado que si declararan que están dispuestos a ser donadores, estarían en peligro de que en ciertas situaciones de emergencia se les deje morir para extraer sus órganos, en lugar de tratar de salvar sus vidas (Weiss, 2003). La desconfianza que impera en algunos países llega al punto de que algunas personas no desean donar por miedo al tráfico de órganos (Frates y Garcia, 2002; Marván et al., 2017).

Por el contrario, también se han revisado algunos factores que favorecen la disposición a donar órganos. Hill (2016), por ejemplo, estudió algunas variables de personalidad, hallando que la amabilidad es un predictor de actitudes positivas hacia la donación, y que el altruismo desempeña un papel mediador en esta relación. Cabe destacar que otros autores ya habían asociado el altruismo con las actitudes positivas hacia la donación (Wakefield, Watts, Homewood y Meiser, 2010). Por otra parte, la disposición a donar también se ha asociado con niveles altos de empatía (Wilczek-Rużyczka, Milaniak, Przybyłowski, Wierzbicki y Sadowski, 2014), que es una habilidad social que permite anticiparse a lo que otros piensan o sienten y que suele preceder a las conductas prosociales (Gaviria, Cuadrado y López, 2009).

Como se ha evidenciado, la disposición a donar órganos depende de diversos factores psico- 
sociales, lo que hace necesario el estudio de las posturas hacia la donación, entendiendo por estas al conjunto de creencias y cogniciones dotadas de una carga afectiva en favor o en contra de un objeto social, mismas que predisponen a comportarse de una determinada manera frente a aquel (Forgas, Cooper y Crano, 2010). De esta manera, se podrá avanzar en las razones de la escasez de órganos; particularmente en países como México, en los que la tasa de donación es muy reducida, para así poner en práctica medidas que fomenten la cultura de la donación, tomando en cuenta las premisas particulares que imperan en la población. Parece apropiado, por consiguiente, analizar las actitudes hacia la donación de órganos utilizando una escala Likert, que se caracteriza por ofrecer una amplia gama de respuestas, bajo la premisa de considerar las actitudes como un continuo que va de lo favorable a lo desfavorable. A diferencia de las preguntas dicotómicas, la escala Likert permite conocer el grado de conformidad del encuestado con las afirmaciones que se le proponen (Sullivan y Artino, 2013). Al hacer una revisión crítica de los instrumentos dirigidos a la población general que se han empleado en distintos países latinoamericanos, se ha visto que dichos instrumentos (cf. Bustamante y Villarreal, 2008; López et al., 2016; Rodríguez, Pérez, Rojas, Reyes y Méndez, 2004) no poseen el rigor estadístico que se sugiere en la actualidad para elaborar instrumentos psicométricamente robustos. Por lo tanto, el objetivo del presente estudio -aprobado previamente por un comité revisor del Instituto de Investigaciones Psicológicas de la Universidad Veracruzana, encargado de revisar los aspectos metodológicos y éticos del protocolo- fue desarrollar y validar una escala Likert para medir las actitudes hacia la donación de órganos postmortem en la población general.

\section{MÉTODO}

\section{Participantes}

La muestra inicial estuvo conformada por 845 mexicanos -número que excede el criterio recomendado para llevar a cabo análisis psicométricos (Bea- vers et al., 2013; Ferrando y Anguiano, 2010)-, de los cuales 412 fueron hombres y 433 mujeres, de quienes se balanceó la edad y la escolaridad. La edad de los participantes osciló entre 20 y 85 años, en tanto que su escolaridad varió desde la educación básica hasta la educación superior. Los participantes vivían en diferentes ciudades, en 31 de los 32 estados del país. La mayoría se declaró católico o practicante de otras religiones cristianas (86\%), lo que es consistente con la prevalencia de esta religión en la población mexicana. Para realizar el análisis factorial confirmatorio se agregaron 184 voluntarios con las mismas características que los de la muestra inicial, quedando así un total de 1,029 participantes.

Se reclutó a la mayoría de los participantes por solicitud directa en lugares públicos, como parques, plazas comerciales, centrales camioneras y mercados. A algunos de los adultos mayores se les reclutó en asilos y casas de día, así como en un sindicato de jubilados. El criterio de inclusión fue la mayoría de edad (18 años o más) y que los participantes tuvieran conocimiento de la donación de órganos para trasplantes, lo que se constató haciendo preguntas tales como si sabían qué era y para qué servía la donación de órganos. El criterio de exclusión fue que no completaran el cuestionario. De las 1,334 personas a las que se invitó a participar y que cumplían los criterios de inclusión, 1,116 de ellas aceptaron (83.7\%), y se excluyó a 87 porque no completaron el cuestionario.

\section{Instrumento}

Para diseñar el cuestionario de Actitudes hacia la Donación de Órganos Postmortem (ADOP en lo sucesivo) se elaboraron 55 reactivos tipo Likert de cinco puntos (de 1 = totalmente desacuerdo, a $5=$ totalmente de acuerdo). La mayoría de los reactivos se elaboraron con base en los resultados de un estudio previo realizado con 439 voluntarios con edades entre los 20 y 80 años, y de diferentes niveles educativos, utilizando en el diseño la técnica de redes semánticas naturales. Otros reactivos se construyeron a partir de una revisión de la literatura sobre el tópico.

Se pidió a cinco expertos (dos médicos, dos psicólogos y un experto en bioética) que evalua- 
ran cada reactivo con base en los siguientes criterios: a) congruencia de los reactivos con la literatura acerca del tema (relevancia); b) posibilidad de sesgo de los reactivos, esto es, si la estructura o la redacción de los reactivos podían influir en la respuesta de las personas, y c) consistencia de la redacción con la forma de expresión de la cultura mexicana. Como resultado, se hicieron algunos cambios en varios reactivos. Después, se pidió a otros 18 jueces (seis psicólogos, siete médicos y cinco enfermeras) que calificaran cada reactivo como "esencial", "útil pero no esencial" o "no necesario", para lo cual se usó la fórmula de Lawshe para calcular la validez de contenido (Cohen y Swerdlik, 2000). Se descartaron los reactivos que no alcanzaron los valores mínimos requeridos, quedando así conformado el instrumento por 35 reactivos. El cuestionario se piloteó con 20 personas de diferentes edades y escolaridades, a quienes se reclutó por solicitud directa en lugares públicos, solicitándoles que respondieran todas las preguntas y que discutieran con el investigador sobre cualquier reactivo que consideraran difícil de entender. Con base en los comentarios, se revisaron algunos reactivos, tras de lo cual se aplicó el cuestionario resultante a los 845 adultos, muestra descrita anteriormente.

Antes de que los participantes respondieran el ADOP, se les pidió que contestaran algunas preguntas sociodemográficas en una hoja independiente, e indicaran si estaban dispuestos a donar sus órganos al morir.

\section{Procedimiento}

Una vez que una persona aceptaba participar en el estudio, y tras verificar que cumplía con los criterios de inclusión se acordaba el lugar, día y hora para aplicarle la encuesta, instruyéndola para que no pusiera ninguna marca de identificación en los cuestionarios y aclarándole que la información que proporcionara se mantendría en el anonimato. También se le subrayaba el hecho de que no había respuestas correctas o incorrectas, y que podía retirarse en cualquier momento si decidía no completar el cuestionario. Finalmente, también se le mencionó al participante que el estudio no tenía como objetivo reunir información individual y que los cuestionarios se combinarían con los de otros participantes para poder analizarlos en conjunto. Al final, se obtuvo el consentimiento informado de todos los participantes.

\section{Análisis de datos}

Mediante el método de grupos extremos, se analizó la capacidad discriminatoria de los reactivos comparando las puntuaciones de cada reactivo entre el 25\% de los participantes con los puntajes más altos y el $25 \%$ con los puntajes más bajos en el cuestionario completo. Para ello, se utilizó la prueba $t$ de Student para muestras independientes.

El análisis factorial exploratorio se llevó a cabo mediante el procedimiento de extracción de mínimos cuadrados no ponderados (Flora, LaBrish y Chalmers, 2012), y se hizo una rotación Varimax (Kaiser). Este procedimiento se repitió tantas veces como fue necesario para obtener una estructura factorial estable. Se eliminaron los reactivos que tuvieron una carga factorial menor a $0.35 \mathrm{o}$ que no estaban relacionados conceptualmente con el factor.

El análisis factorial confirmatorio se realizó usando el procedimiento de estimación de máxima verosimilitud. Los estándares mínimos de los índices de ajuste que se consideraron fueron, a saber: $\mathrm{x}^{2} / \mathrm{gl}<3$; la raíz cuadrada media del error de aproximación (RMSEA), con su intervalo de confianza asociado de 90\%; el índice de ajuste comparativo (CFI), y el índice de ajuste de Tucker-Lewis (Byrne, 2010; Hu y Bentler, 1999). Además, se empleó el estadístico de Hoelter para comprobar si el tamaño de la muestra proporcionaba una estimación adecuada del ajuste del modelo, para lo cual se determinó un punto mínimo de corte de 200 (Byrne, 2010).

Se consideró el efecto techo/piso cuando más del 15\% de los encuestados obtuviera las puntuaciones más bajas o más altas posibles en el ADOP. La consistencia interna se probó utilizando el coeficiente alfa de Cronbach, y también se calcularon los coeficientes de correlación entre los factores obtenidos por medio del análisis factorial.

Finalmente, se compararon los puntajes del ADOP entre los participantes que estuvieron dispuestos a ser donadores de órganos después de la muerte y los que no, utilizando para este propósito la prueba $t$ de Student para muestras independientes. 


\section{RESULTADOS}

El análisis de grupos extremos mostró que todos los reactivos tenían una adecuada capacidad discriminatoria, ya que sí fueron significativas las diferencias entre los participantes que obtuvieron los puntajes más altos y más bajos en el cuestionario (todos los valores de probabilidad menores de 0.05 se consideraron significativos).

\section{Análisis factorial exploratorio}

Se llevaron a cabo tres rondas de análisis factorial exploratorio; en la primera se eliminaron ocho reac- tivos, y en la segunda uno. El análisis final sugirió un instrumento de 26 reactivos con tres factores claros e interpretables que representaron $40.12 \%$ de la varianza común (sin errores de medición). Dichos factores se denominaron "Actitudes favorables" cuyos reactivos se refieren a la importancia de la donación de órganos; "Actitudes desfavorables", con reactivos que sugieren rechazo o actitudes negativas hacia la donación de órganos, y "Actitudes de desconfianza" con reactivos que reflejan falta de confianza, principalmente en el manejo ético de todo el proceso de donación y trasplante. La estructura factorial se presenta en la Tabla 1.

Tabla 1. Cargas factoriales del cuestionario obtenidas en el análisis factorial exploratorio.

\begin{tabular}{|c|c|c|c|}
\hline \multirow[b]{2}{*}{ Reactivos } & \multicolumn{3}{|c|}{ Solución de tres factores } \\
\hline & $\begin{array}{c}\text { Actitudes } \\
\text { favorables }\end{array}$ & \begin{tabular}{c|c} 
Actitudes \\
desfavorables
\end{tabular} & $\begin{array}{l}\text { Actitudes de } \\
\text { desconfianza }\end{array}$ \\
\hline Al donar órganos se da esperanza a otras personas. & .766 & & \\
\hline Al morir, nuestros órganos pueden servir para que otras personas recuperen su salud. & .738 & & \\
\hline El donar órganos ayuda a mejorar la calidad de vida de otros. & .699 & & \\
\hline Es satisfactorio que los órganos puedan servir a otros, aunque estos sean desconocidos. & .671 & & \\
\hline La donación de órganos al morir es positiva para la sociedad. & .631 & & \\
\hline Es necesario fomentar la cultura de donación de órganos. & .629 & & \\
\hline Con la donación de órganos se puede beneficiar a muchas personas. & .625 & & \\
\hline El que alguien done sus órganos al morir es una acción solidaria. & .609 & & \\
\hline La donación de órganos es un acto de amor al prójimo. & .601 & & \\
\hline $\begin{array}{l}\text { Si antes de morir un familiar decide donar sus órganos, yo haría lo necesario para } \\
\text { que se lleve a cabo la donación. }\end{array}$ & .501 & & \\
\hline La donación de órganos es una acción desinteresada. & .496 & & \\
\hline El donar órganos es un regalo invaluable. & .477 & & \\
\hline La donación de órganos es una falta de respeto para el cuerpo del que dona. & & .697 & \\
\hline Donar órganos interrumpe el proceso natural de morir. & & .683 & \\
\hline Siento que si mis órganos se donan cuando muera, no descansaría en paz. & & .638 & \\
\hline Me da miedo que al morir se donen mis órganos. & & .622 & \\
\hline Me opongo a donar mis órganos porque estos pertenecen a una sola persona. & & .604 & \\
\hline Es desagradable pensar que al donar órganos el cuerpo queda incompleto. & & .572 & \\
\hline La donación de órganos va en contra de mi religión. & & .460 & \\
\hline $\begin{array}{l}\text { Cuando se está triste por la muerte de un familiar, es una falta de respeto pedir } \\
\text { que sus órganos se donen. }\end{array}$ & & .452 & \\
\hline Prolongar la vida por medio de la donación de órganos es artificial. & & .365 & \\
\hline Me opongo a donar mis órganos al morir porque hay mucha corrupción en el proceso. & & & .560 \\
\hline Desconfío de las instituciones en las que se realizan trasplantes de órganos. & & & .524 \\
\hline Me angustia pensar que si tengo un accidente y soy donador sea mal atendido. & & & .522 \\
\hline $\begin{array}{l}\text { Me preocupa no estar realmente muerto cuando se haga la cirugía para la dona- } \\
\text { ción de órganos. }\end{array}$ & & & .443 \\
\hline Con la donación de órganos se hace negocio. & & & .395 \\
\hline
\end{tabular}




\section{Análisis factorial confirmatorio}

Los índices de bondad de ajuste para el ADOP fueron subóptimos en este punto, en virtud de que los índices de modificación propusieron cuatro covarianzas entre los términos de error de ocho reactivos. La evaluación del contenido de tales reactivos mostró superposición, por lo que se elimi- naron los cinco con cargas factoriales más bajas, realizándose otro análisis confirmatorio. El modelo final mostró índices de ajuste óptimos: $\mathrm{x}^{2} / \mathrm{gl}=$ 2.64, RMSEA $=.040$, CFI $=.959$, y NNFI $=.935$. Finalmente, el estadístico de Hoelter fue de 459 $(\alpha=0.05)$, lo que permite concluir que el tamaño de la muestra fue adecuado. La Figura 1 muestra la estructura factorial definitiva.

Figura 1. Análisis factorial confirmatorio. Diagrama de la solución de tres factores con las cargas factoriales estandarizadas de cada reactivo y los valores específicos de correlación.

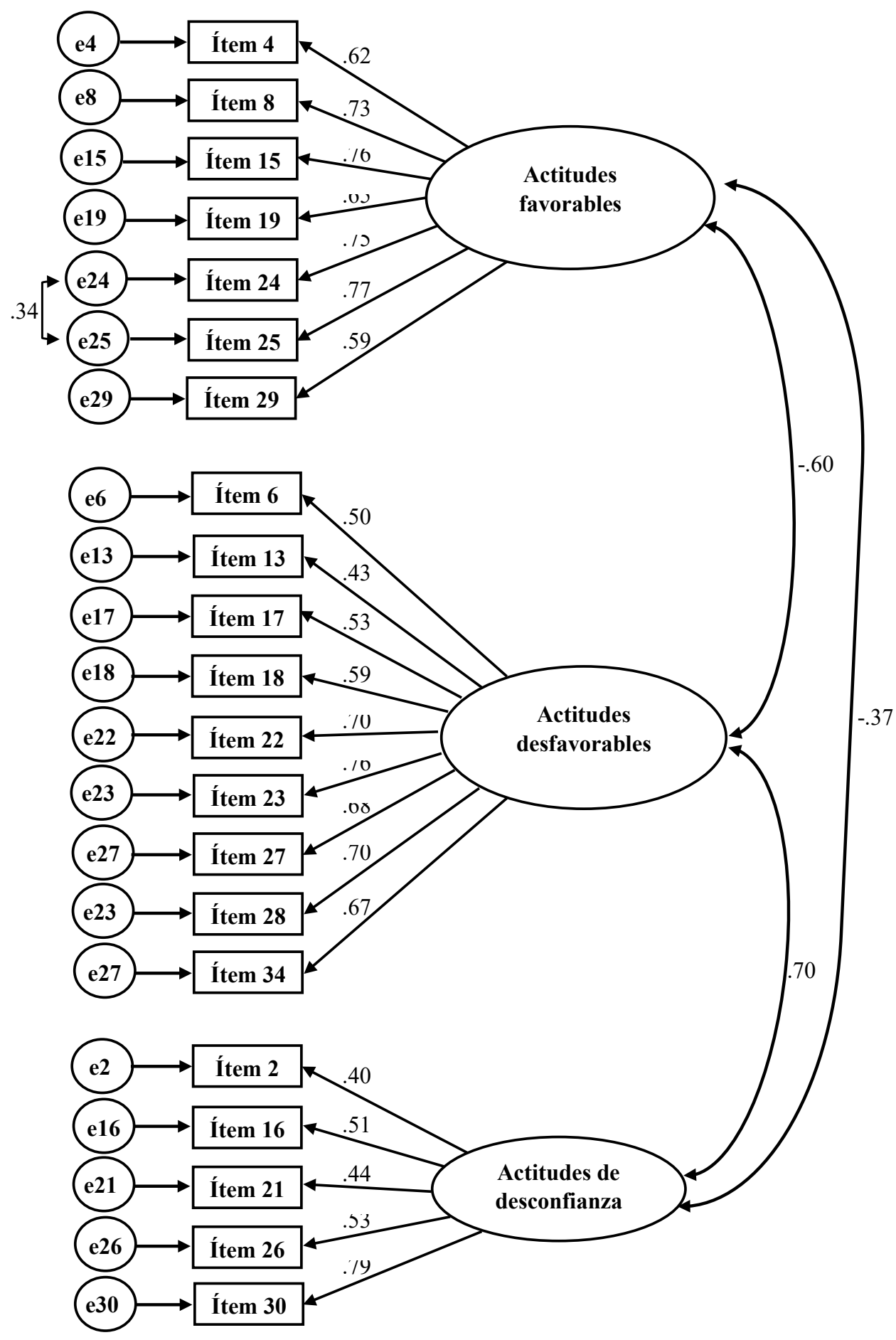




\section{Efecto suelo/techo}

No se encontró efecto suelo/techo debido a que ningún participante obtuvo las puntuaciones más bajas o más altas posibles del ADOP.

\section{Confiabilidad}

El factor de Actitudes favorables (siete reactivos) obtuvo un coeficiente alfa de Cronbach de 0.85 , el de Actitudes desfavorables (nueve reactivos), de 0.84 , y el de Actitudes de desconfianza (cinco reactivos), de 0.68 . El instrumento total con 21 reactivos tuvo un coeficiente de 0.87 , lo que indica una buena confiabilidad.

\section{Correlaciones entre factores}

El factor Actitudes desfavorables mostró una correlación con los otros dos factores en direcciones opuestas: una correlación negativa con el factor Actitudes favorables $(\mathrm{r}=-.60)$ y una positiva con el factor Actitudes de desconfianza $(r=.70)$. Por último, el factor Actitudes favorables tuvo una correlación negativa con el de Actitudes de desconfianza $(r=-.37)$. Cabe señalar que todas las correlaciones fueron significativas $(p<.001)$.

\section{Resultados adicionales}

Hubo 773 participantes (75\%) que estaban dispuestos a donar sus órganos al morir, 237 que no lo estaban (23\%), y 19 (2\%) que no estaban seguros de lo que querían. Comparando los puntajes del ADOP entre los participantes que estaban dispuestos a donar y los que no lo estaban, se encontraron diferencias significativas en los tres factores. Los participantes que estuvieron dispuestos a donar mostraron más actitudes favorables $(\mathrm{M}=4.79$, D.E. $=.37 \mathrm{y} \mathrm{M}=4.18$, D.E. $=.97$ respectivamente; $\mathrm{t}=14.59, \mathrm{gl}=1008, p<.0001)$; así como menos actitudes desfavorables $(\mathrm{M}=1.67$, D.E. $=.71 \mathrm{y}$ $\mathrm{M}=2.81$, D.E. $=1.13$, respectivamente; $\mathrm{t}=148.44$, $\mathrm{gl}=1008, p<.0001)$ y de desconfianza $(\mathrm{M}=2.94$, D.E. $=.99$ y $\mathrm{M}=3.76$, D.E. $=1.01$, respectivamente; $\mathrm{t}=11.15, \mathrm{gl}=1008, p<.0001)$.

\section{DISCUSIÓN}

El propósito de este estudio fue desarrollar una nueva escala para medir actitudes hacia la donación de órganos postmortem. La escala resultante posee propiedades psicométricas confiables, así como una estructura factorial clara e interpretable. Los análisis factoriales, tanto el exploratorio como el confirmatorio, indicaron la existencia de tres factores: Actitudes favorables, Actitudes desfavorables y Actitudes de desconfianza.

Una virtud del ADOP es que los tres factores que lo componen distinguen entre los individuos que están dispuestos a donar sus órganos después de la muerte y los que no lo están. Como es de esperarse, los participantes que estaban dispuestos a donar fueron los más propensos a mostrar actitudes favorables, y los que menos lo estaban presentaban actitudes desfavorables y de desconfianza.

Llama la atención el hecho de que, además de las actitudes favorables y desfavorables, el análisis factorial haya arrojado una estructura con un tercer factor, cuyos reactivos implican desconfianza. Este resultado no sorprende porque, según el Índice de Percepción de la Corrupción publicado por Transparency International (2016), México obtuvo un puntaje de 30/100 (las puntuaciones más bajas indican la mayor percepción de corrupción), siendo 38.5 el promedio de los países latinoamericanos. Sería conveniente comparar los resultados del factor Actitudes de desconfianza del ADOP entre países con diferentes niveles de percepción de corrupción.

Un resultado notable fue que la mayoría de los participantes dijo estar dispuesta a donar sus órganos al morir, lo que es consistente con el hecho de que los puntajes del factor Actitudes positivas fueran más altos que los de Actitudes desfavorables y de Desconfianza, lo que parece contradecir la reducida tasa de donación en el país. Lo anterior puede tener algunas explicaciones: $a$ ) Que, de acuerdo con Van den Berg, Manstead, Van der Pligt y Wigboldus (2005), el componente afectivo de las actitudes es el que realmente predice la toma de decisiones importantes para donar órganos; y que el factor Actitudes desfavorables del ADOP es el que tiene la mayor proporción de reactivos que tienden a evaluar el aspecto afectivo 
de las actitudes, mientras que el factor Actitudes favorables es el que tiene la mayor proporción de reactivos sobre el componente cognitivo; es decir, a pesar de que los participantes reconocen la importancia de la donación de órganos (aspecto cognitivo), hay elementos afectivos que la pueden impedir. b) Que la tendencia de los individuos es dar respuestas socialmente aceptables (Sander y Miller, 2005; Wong, 2011); c) Que el hecho de estar dispuesto a ser donador no implica necesariamente que la donación se efectúe, pues existen otras variables que pueden interferir en el proceso, como las circunstancias de la muerte del posible donador o la negativa de la familia.

La baja tasa de consentimiento de las familias de los donadores potenciales es un factor que influye de manera importante en la escasez de transplante de órganos (Martínez et al., 2001; Siminoff, Gordon, Hewlett y Arnold, 2001). El ADOP contiene dos reactivos relacionados con los familiares: "Si antes de morir un familiar decide donar sus órganos, yo haría lo necesario para que se lleve a cabo esa donación" y "Cuando se está triste por la muerte de un familiar, es una falta de respeto pedir que sus órganos se donen". La mayoría de los participantes ( $87 \%$ ) estuvo de acuerdo con el primer reactivo, pero casi la mitad (43\%) también estuvo de acuerdo con el segundo. En otras palabras, parece ser que, aunque en principio los familiares estarían de acuerdo en donar los órganos de su consanguíneo, sería difícil para ellos hablar sobre ese tópico en el momento preciso en que la donación es viable. Dado que es importante realizar más investigaciones al respecto, sería útil elaborar una nueva versión del ADOP dirigida a las familias de posibles donadores, misma que podría ser utilizada por los profesionales de la salud para diseñar estrategas de intervención apropiadas.

También es imprescindible conocer las actitudes de los profesionales de la salud, hacia la donación de órganos postmortem, lo que podría llevarse a cabo utilizando el ADOP. La importancia de ello radica en que dichos expertos son los primeros en establecer una relación con la familia del potencial donador (Essman y Thornton, 2006), $\mathrm{y}$ en que sus actitudes son esenciales para crear y promover un ambiente que influya positivamente en la donación (Marqués, Ortiz, Sánchez, Soto y Torres, 2013).
Por otro lado, se ha demostrado que las campañas para promover la donación de órganos favorecen considerablemente las actitudes y las discusiones familiares sobre el tema, además de que aumentan el número de personas que señalan expresamente ser donadoras de órganos (Breitkopf, 2009). El ADOP podría utilizarse para diseñar y evaluar campañas a fin de que las mismas disipen ideas erróneas, disminuyan las actitudes desfavorables y proporcionen un conocimiento preciso y actualizado sobre la donación de órganos, teniendo en cuenta las creencias y actitudes de las personas, al igual que su cultura.

Algunos investigadores pueden utilizar el ADOP con el fin de comprender mejor algunos factores psicosociales que pueden influir en la aceptación o el rechazo de donar órganos postmortem. También, puede ser útil para estudiar poblaciones diferentes dentro de una misma cultura y para hacer comparaciones interculturales. Asimismo, parece importante explorar la relación entre las puntuaciones del ADOP y diversos factores que contribuyen a la compresión de esta cuestión. Entre estos factores se podrían incluir el duelo, ciertas variables de personalidad, el imaginario social, los conocimientos y la afiliación religiosa, entre otros.

Por último, estos resultados deben interpretarse en el contexto de las limitaciones del estudio. En primer lugar, a pesar de que los participantes vivían en diferentes estados del país, la muestra por conveniencia no es representativa de la población nacional; por lo tanto, los hallazgos solamente pueden generalizarse de forma limitada. Parece importante comparar en investigaciones futuras las características psicométricas del ADOP entre diferentes muestras según su nivel educativo, edad, etnia, religión o lugar de residencia. En segundo lugar, el factor Actitudes de desconfianza tuvo una confiabilidad marginal, la cual podría explicarse por el hecho de que está compuesto únicamente por cinco reactivos (Anastasi y Urbina, 1997); sin embargo, los presentes autores decidieron retenerlo debido a que la desconfianza en el manejo del proceso de donación y trasplante de órganos es un fenómeno generalizado y representa una barrera importante para la donación de órganos, particularmente en nuestro país. 
En conclusión, el ADOP demostró ser un instrumento válido y confiable que puede ser empleado por profesionales de la salud e investigadores para evaluar cómo es que los individuos conceptúan la donación de órganos.

\section{REFERENCIAS}

Anastasi, A. y Urbina, S. (1997). Psychological testing (7th ed). Hoboken, NJ: Pearson.

Beavers, S.A., Lounsbury, W.J., Richards, K.J., Huck, W.S., Skolits, J.G. y Esquivel, L.S. (2013). Practical considerations for using exploratory factor analysis in educational research. Practical Assessment, Research \& Evaluation, $18,1-13$.

Breitkopf, C.R. (2009). Attitudes, beliefs and behaviors surrounding organ donation among Hispanic women. Current Opinion in Organ Transplantation, 14, 191-195. doi: 10.1097/MOT.0b013e328329255c.

Bruzzone, P. (2008). Religious aspects of organ transplantation. Transplantation Proceedings, 40, 1064-1067. doi: 10.1016/j. transproceed.2008.03.049.

Bustamante U., M. y Villarreal N., P. (2008). Motivación a la donación de órganos en Chile. Panorama Socioeconomico, 26, 86-97.

Byrne, B. (2010). Structural equation modeling with AMOS. Basic concepts, application, and programming (2nd ed.). New York: Routledge.

Cohen, R.J. y Swerdlik, M.E. (2000). Pruebas y evaluación psicológicas: Introducción a las pruebas y a la medición. (4a ed.). Mexico: McGraw-Hill.

Council of Europe (2016). International figures on donation and transplantation 2015. Newsletter Trasplant, 21, 3-61. Recuperado de http://www.ont.es/publicaciones/Documents/NEWSLETTER\%202016\%202.pdf.

Essman, C. y Thornton, J. (2006). Assessing medical student knowledge, attitudes, and behaviors regarding organ donation. Transplantation Proceedings, 38, 2745-2750. doi: 10.1016/j.transproceed.2006.08.127.

Ferrando, P. y Anguiano C., C. (2010). El análisis factorial como técnica de investigación en psicología. Papeles del Psicólogo, 31, 18-33. Recuperado de http://www.papelesdelpsicologo.es/vernumero.asp? ID = 1793 .

Findlater, C. y Thomson, E.M. (2015). Organ donation and management of the potential organ donor. Anaesthesia and Intensive Care Medicine, 16, 315-320. doi: http://dx.doi.org/10.1016/j.mpaic.2015.04.013.

Flora, D.B., LaBrish, C. y Chalmers, R.P. (2012). Old and new ideas data screening and assumption testing for exploratory and confirmatory factor analysis. Frontiers in Psychology, 3, 1-21. doi: 10.3389/fpsyg.2012.00055.

Forgas, J., Cooper, J. y Crano, W. (2010). The psychology of attitudes and attitude change. Sidney (Australia): Psychology Press. Frates, J. y Garcia, B.G. (2002). Hispanic perceptions of organ donation. Progress in Transplantation, 12, 169-175.

Gaviria S., E., Cuadrado G., I. y López S., M. (2009). Introducción a la psicología social. Alcorcón (España): Sanz y Torres.

Gómez R., K., Ballena L., J. y León J., F. (2016). Mitos sobre la donación de órganos en personal de salud, potenciales receptores y familiares de potenciales donantes de un hospital peruano: Estudio cualitativo. Revista Peruana de Medicina Experimental y Salud Pública, 33, 83-91. doi: 10.17843/rpmesp.2016.331.2011.

Güden, E., Çetinkaya, F. y Naçar, M. (2013). Attitudes and behaviors regarding organ donation: A study on officials of religion in Turkey. Journal of Religion and Health, 52, 439-449. doi: 10.1007/s10943-011-9490-8.

Hill, E.M. (2016). Posthumous organ donation attitudes, intentions to donate, and organ donor status: Examining the role of the big five personality dimensions and altruism. Personality and Individual Differences, 88, 182-186. doi: 10.1016/j. paid.2015.09.021.

Hu, L.T. y Bentler, P.M. (1999). Cutoff criteria for fit indexes in covariance structure analysis: Conventional criteria versus new alternatives. Structural Equation Modeling: A Multidisciplinary Journal, 6, 1-55. doi: 10.1080/10705519909540118.

López F., R., Ramírez O., R., Ortiz A., F.I., Rodríguez J., J., Ramírez O., A., Camarena R., H. y Colio M., M. (2016). Attitudes toward organ donation and transplantation in Guanajuato, Mexico. Transplantation Proceedings, 48(2), 556-558. Recuperado de https://doi.org/10.1016/j.transproceed.2016.02.028.

Lozano R., G., Liberman S., S., Ito R., M. y Andrade P., P. (2005). Conocimientos y creencias acerca de la donación de órganos y tejidos en la Ciudad de México. Psicología y Salud, 15(1), 77-83.

Marqués L., J., Ortiz V., N., Sánchez, M., Soto A., O. y Torres, E. (2013). Knowledge of and attitudes toward organ donation: A survey of medical students in Puerto Rico. Puerto Rico Health Sciences Journal, 32, 187-193.

Martínez J., M., López J., S., Martín, A., Martín, M.J., Scandroglio, B. y Martín J., M. (2001). Organ donation and family decision-making within the Spanish donation system. Social Science \& Medicine, 53, 405-421. doi: 10.1016/S02779536(00)00345-2. 
Marván, M.L., Álvarez del Río, A., Jasso, K. y Santillán D., P. (2027). Psychosocial barriers associated with organ donation in Mexico, Clinical Transplantation, 31. Recuperado de http://onlinelibrary.wiley.com/doi/10.1111/ctr.13112/full en línea. doi: $10.1111 /$ ctr.13112.

Randhawa, G. y Neuberger, J. (2016). Role of religion in organ donation. Development of the United Kingdom Faith and Organ Donation Action Plan. Transplantation Proceedings, 48(3), 689-694.

Rodríguez O., G., Pérez F., C., Rojas R., M., Reyes L., I. y Méndez R., I. (2004). Trasplante de órganos y tejidos: creencias, actitudes e intenciones conductuales en la población mexicana. En G. Rodríguez (Coord.): Medicina conductual en México. México: Miguel Ángel Porrúa.

Sander, S.L. y Miller, B.K. (2005). Public knowledge and attitudes regarding organ and tissue donation: an analysis of the northwest Ohio community. Patient Education and Counseling, 58(2), 154-163.

Siminoff, L.A., Gordon, N., Hewlett, J. y Arnold, R.M. (2001). Factors influencing families' consent for donation of solid organs for transplantation. Journal of American Medical Association, 286, 71-77. doi: 10.1001/jama.286.1.71.

Sullivan, G.M. y Artino, A.R. (2013). Analyzing and interpreting data from Likert-type scales (2013). Journal of Graduate Medical Education, 5(4), 541-542.

Transparency International (2016). Corruptions perceptions index 2015. Recuperado de https://www.transparency.org/cpi2015/ \#results-table.

Van den Berg, H., Manstead, A., Van der Pligt, J. y Wigboldus, D. (2005). The role of affect in attitudes toward organ donation and donor- relevant decisions. Psychology Health, 20, 789-802.

Wakefield, C.E., Watts, K.J., Homewood, J. y Meiser, B. (2010). Attitudes toward organ donation and donor behavior: A review of the international literature. Progress in Transplantation, 20, 380-391. doi: 10.7182/prtr.20.4.p54651601pg80183.

Weiss, R. (2003). Boosting organ donation among Hispanics. Health Progress, January-February, 13-14.

Wilczek-Rużyczka, E., Milaniak, I., Przybyłowski, P., Wierzbicki, K. y Sadowski, J. (2014). Influence of empathy, beliefs, attitudes, and demographic variables on willingness to donate organs. Transplantation Proceedings, 46, 2505-2508. doi: 10.1016/j.transproceed.2014.09.024.

World Health Organization (2009). Global glossary of terms and definitions on donation and transplantation. Geneve: WHO. Recuperado de http://www.who.int/transplantation/activities/GlobalGlossaryonDonationTransplantation.pdf?ua $=1$.

World Health Organization (2017). Donation and transplantation. Geneve: WHO. Recuperado de http://www.who.int/transplantation/donation/en/\#.

Wong, L.P. (2011). Knowledge, attitudes, practices and behaviors regarding deceased organ donation and transplantation in Malaysia's multi-ethnic society: A baseline study. Clinical Transplantation, 25, E22- E31. doi: 10.1111/j.1399-0012.2010.01312.x.

Zepeda R., L., García G., G. y Aguirre J., O. (2003). Resultados de una encuesta sobre donación y trasplante de órganos en la zona metropolitana de Guadalajara, México. Salud Pública de México, 45, 54-57. 


\section{APÉNDICE}

\section{Versión final del Cuestionario de Actitudes hacia la Donación de Órganos Postmortem (ADOP)}

A continuación se presentan varias afirmaciones sobre la donación de órganos al morir. Le pedimos que conteste qué tan de acuerdo o desacuerdo está con ellas, siendo 1 "Totalmente en desacuerdo" y 5 "Totalmente de acuerdo". Marque con una X el recuadro correspondiente.

\begin{tabular}{|c|c|c|c|c|c|}
\hline \multirow[t]{2}{*}{ REACTIVOS } & \multirow{2}{*}{ 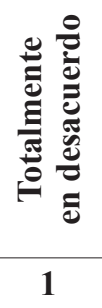 } & & & & \multirow{2}{*}{ 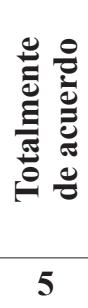 } \\
\hline & & 2 & 3 & 4 & \\
\hline \multicolumn{6}{|l|}{ 1. Es necesario fomentar la cultura de donación de órganos al morir. } \\
\hline \multicolumn{6}{|l|}{ 2. La donación de órganos va en contra de mi religión. } \\
\hline \multicolumn{6}{|l|}{ 3. Me angustia pensar que si tengo un accidente y soy donador sea mal atendido. } \\
\hline \multicolumn{6}{|l|}{ 4. El donar órganos ayuda a mejorar la calidad de vida de otros. } \\
\hline \multicolumn{6}{|l|}{ 5. Prolongar la vida por medio de la donación de órganos es artificial. } \\
\hline \multicolumn{6}{|l|}{ 6. Me da miedo que al morir se donen mis órganos. } \\
\hline \multicolumn{6}{|l|}{ 7. Desconfío de las instituciones en las que se realizan trasplantes de órganos. } \\
\hline \multicolumn{6}{|l|}{ 8. Es satisfactorio que los órganos puedan servir a otros, aunque sean desconocidos. } \\
\hline \multicolumn{6}{|l|}{ 9. Es desagradable pensar que al donar órganos el cuerpo queda incompleto. } \\
\hline \multicolumn{6}{|l|}{ 10. Con la donación de órganos se hace negocio. } \\
\hline \multicolumn{6}{|l|}{$\begin{array}{l}\text { 11. Si un familiar antes de morir decide donar sus órganos, yo haría lo necesario para } \\
\text { que se lleve a cabo la donación. }\end{array}$} \\
\hline \multicolumn{6}{|l|}{$\begin{array}{l}\text { 12. Cuando se está triste por la muerte de un familiar, es una falta de respeto pedir que } \\
\text { sus órganos se donen. }\end{array}$} \\
\hline \multicolumn{6}{|l|}{ 13. Donar órganos interrumpe el proceso natural de morir. } \\
\hline \multicolumn{6}{|l|}{$\begin{array}{l}\text { 14. Me preocupa no estar realmente muerto cuando se haga la cirugía para la donación } \\
\text { de órganos. }\end{array}$} \\
\hline \multicolumn{6}{|l|}{ 15. Al donar órganos se da esperanza a otras personas. } \\
\hline \multicolumn{6}{|l|}{ 16. Me opongo a donar mis órganos porque estos pertenecen a una sola persona. } \\
\hline \multicolumn{6}{|l|}{$\begin{array}{l}\text { 17. Me opongo a donar mis órganos al morir porque hay mucha corrupción en el } \\
\text { proceso. }\end{array}$} \\
\hline \multicolumn{6}{|l|}{$\begin{array}{l}\text { 18. Al morir, nuestros órganos pueden servir para que otras personas recuperen su } \\
\text { salud. }\end{array}$} \\
\hline \multicolumn{6}{|l|}{ 19. La donación de órganos es una falta de respeto para el cuerpo del que dona. } \\
\hline \multicolumn{6}{|l|}{ 20. Siento que si mis órganos se donan cuando muera no descansaría en paz. } \\
\hline 21. La donación de órganos es un acto de amor al prójimo. & & & & & \\
\hline
\end{tabular}

